\title{
Single molecule Raman detection of enkephalin on silver colloidal particles
}

\author{
Katrin Kneipp ${ }^{\text {a,b,c,d,* }}$, Harald Kneipp ${ }^{\text {b,d }}$, Salim Abdali ${ }^{\text {a }}$, Rolf W. Berg ${ }^{\text {e }}$ and Henrik Bohr ${ }^{\text {a }}$ \\ ${ }^{a}$ Quantum Protein Center, Technical University of Denmark, 2800 Kgs. Lyngby, Denmark \\ ${ }^{\mathrm{b}}$ Department of Physics, Technical University Berlin, 10234 Berlin, Germany \\ ${ }^{\mathrm{c}}$ Department of Electrical Engineering and Computer Science, Massachusetts Institute of Technology, \\ Cambridge, MA 02139, USA \\ ${ }^{\mathrm{d}}$ Wellman Center for Photomedicine, Harvard Medical School, Boston, MA 02114, USA \\ ${ }^{\mathrm{e}}$ Department of Chemistry, Technical University of Denmark, $2800 \mathrm{Kgs}$. Lyngby, Denmark
}

\begin{abstract}
Enkephalin, an endogeneous substance in the human brain showing morphine-like biological functions, has been detected at the single molecule level based on the surface-enhanced Raman signal of the ring breathing mode of phenylalanine, which is one building block of the molecule. For enhancing the Raman signal the enkephalin molecules have been attached to silver colloidal cluster structures. The experiments demonstrate that the SERS signal of the strongly enhanced ring breathing vibration of phenylalanine at $1000 \mathrm{~cm}^{-1}$ can be used as "intrinsic marker" for detecting a single enkephalin molecule without using a specific label molecule. The reported result suggests the use of the phenylalanine $1000 \mathrm{~cm}^{-1}$ SERS line as spectroscopic signature for monitoring single proteins containing this amino acid as a building block.
\end{abstract}

Keywords: SERS, single molecules, silver colloids, peptides, proteins, local optical fields, enkephalin

\section{Introduction}

Detecting single molecules, monitoring their location and movement, and identifying their chemical structure is a field of rapidly growing interest. Laser spectroscopic techniques play an important role in developing single molecule tools [1].

Single molecule spectroscopy is particularly appealing in the field of biophysics, biochemistry and medicine [2,3], where in many cases, molecules of interest are available at extremely low concentration level only. Furthermore, the observation of spectroscopic signals and processes at the single molecule level is of basic scientific interest, as it provides insight into the intrinsic properties of a molecule or a process and permits observations without ensemble averaging.

Fluorescence is widely used as an ultrasensitive and single molecule spectroscopic tool in biophysics [2]. To detect and to identify single molecules by fluorescence, in most cases, they must be labeled by fluorescent dye molecules to achieve high enough fluorescence quantum yields and distinguishable spectral properties.

Alternatively to fluorescence, single molecules can be detected based on their surface enhanced Raman scattering (SERS) signal [4-6]. When the target molecule is attached to silver or gold nanostructure, the

\footnotetext{
${ }^{*}$ Corresponding author. E-mail: kneipp@ usa.net.
} 
nonresonant Raman signal can be enhanced up to 14 orders of magnitude and effective Raman cross sections can reach and even exceed fluorescence cross sections of dyes used as fluorescence labels [7].

Ideally, one would like to have a spectroscopic tool, which detects single molecules and simultaneously identifies their chemical structures. A SERS spectrum, containing different vibrational modes, provides high structural information content on a molecule and something like its "fingerprint". At present, SERS is the only way to detect a single molecule and simultaneously identify its chemical structure.

Also measuring only one typical SERS line of the target molecule and using this Raman line as a spectroscopic signature for the specific molecule is a useful tool for detecting a known molecule and for monitoring its distribution without using fluorescence labels. Limitations of SERS spectroscopy are attributed to the fact that target molecules have to be attached to silver or gold nanostructures, where chemically inert nanometer gold particles might be the favorite choice for biomedical applications.

In addition to avoiding the use of fluorescence labels, SERS detection can provide also other advantages compared to using fluorescence signals. Under biologically relevant conditions, such as in solutions and at room temperature, fluorescence signals are very broad spectral signatures, typically 400 $800 \mathrm{~cm}^{-1}$ in widths. Compared to a fluorescence band, Raman scattering results in spectrally narrow lines typically $10-20 \mathrm{~cm}^{-1}$ in width, even if inhomogeneous broadening occurs (see our discussion on homogeneous and inhomogeneous broadening in the text below). As a further advantage, SERS detection is insensitive to photo bleaching and self-quenching, since the effect works also well as nonresonant process, where the excitation laser energy does not meet an electronic transition in the target molecule.

In this work, we apply SERS on colloidal silver clusters to enkephalin molecules. Enkephalin has been isolated from pig brain relatively late in 1974 [8]. The substance can be found in the brain and the spinal cord where it has a morphine-like function as pain killer. Enkephalin is a mixtures of two pentapeptides, [Leu]enkephalin and [Met]enkephalin. During the last years, several experimental and theoretical studies were carried out on enkephalin including X-ray-, NMR-, Raman- and vibrational circular dichroism (VCD) measurements [9-11]. Recently we performed FT-Raman studies on [Leu]enkephalin and [Met]enkephalin in solution, where we studied the effect the of $\mathrm{pH}$-value on the conformational states of the two molecules [11].

In this SERS study we detect [Leu]enkephalin and [Met]enkephalin at the single molecule level based on the strongly enhanced ring breathing mode of phenylalanine, which is a building block of both pentapeptides.

\section{Experimental}

Colloidal silver solution has been prepared by reduction of silver nitrate by sodium citrate as it has been described in [12]. Most of the colloidal silver particles formed during this process are nearly spheres at sizes between 15 and $40 \mathrm{~nm}$. These particles form small colloidal clusters between 150 and $300 \mathrm{~nm}$ dimension as shown in the electron microscopic view in Fig. $1 \mathrm{~b}$. Addition of $10^{-1} \mathrm{M}$ sodium citrate favors additionally aggregation of the colloidal silver particles resulting in colloidal clusters in the micron size range, which can be seen in a light microscope.

The pentapeptides [Leu]enkephalin (Tyr-Gly-Gly-Phe-Leu) and [Met]enkephalin (Tyr-Gly-Gly-PheMet) were purchased from Bavhem as powder. Aqueous solutions of the substances have been added to the colloidal silver solution resulting in concentrations between $10^{-8}$ and $10^{-11} \mathrm{M}$ in the final sample solution. $5 \mu \mathrm{l}$ of these sample solutions have been dried on a microscopic cover slide covering an area of approximately $2.5 \times 10^{-5} \mathrm{~m}^{2}$. 


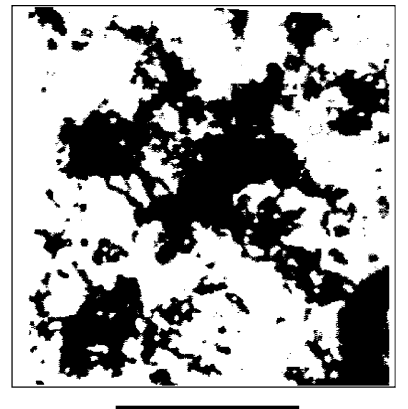

a)

$20 \mu \mathrm{m}$

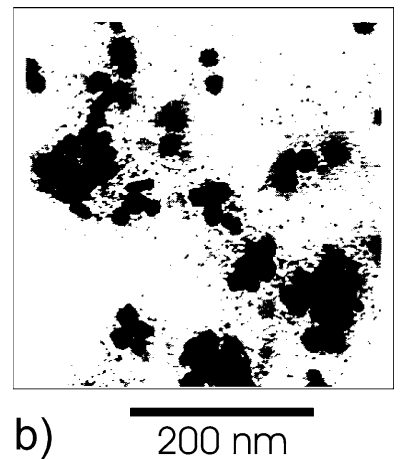

Fig. 1. Light- (a) and electron- (b) microscopic views of typical colloidal silver particles at different aggregation stages providing SERS enhancement levels up to 14 orders of magnitude.

SERS measurements were carried out using $514.5 \mathrm{~nm}$ excitation applying laser powers between 3-5 mW. The excitation laser light was focused by a microscope objective to a spot size of about 1 micron. The same microscope objective was also used for collecting the Raman scattered light. A single stage spectrograph and a CCD detector were used for spectral dispersion and collection of the scattered light. Good signal to noise SERS spectra were measured in one second collection time. For scanning and looking for single target molecules, the sample was moved in $1 \mu \mathrm{m}$ raster steps under the laser focus. When a signal was found, measurements in time sequence were performed at that point of the sample.

\section{Results and discussion}

After evaporation, the colloidal sample solutions forms silver cluster structures on the cover slide as shown for two size dimensions ( $20 \mu \mathrm{m}$ scale and $200 \mathrm{~nm}$ scale) in Fig. 1. In general, such fractal colloidal cluster structures show a very inhomogeneous distribution of the optical fields on their surface resulting in so-called "hot spots", where nonresonant SERS enhancement factors can reach fourteen orders of magnitude $[5,7,13,14]$.

Figure 2a shows typical unprocessed SERS spectra from [Met]enkephalin on silver colloidal clusters. For comparison, Fig. 2b shows "normal" Raman spectra of [Met]enkephalin and [Leu]enkephalin measured at $1064 \mathrm{~nm}$ excitation in a FT-transform Raman system. Figure $2 \mathrm{c}$ gives the structural formula of both compounds. The strongest Raman line in both normal and surface-enhanced spectra appears at $1000 \mathrm{~cm}^{-1}$ and can be ascribed to the ring breathing mode of phenylalanine. The Raman spectra are discussed in more detail in [11]. Note that the normal Raman spectra are measured from at least $10^{10}$ times more enkephalin molecules than used in SERS experiments. Spectra in Fig. 2a have been measured from two places of a SERS sample, which have approximately 1000 [Met]enkephalin molecules in the probed area of approximately $1 \mu \mathrm{m}^{2}$. This number does not mean that 1000 molecules contribute to the SERS signal. Due to the very small area of "hot spots" within the focal spot it is very likely that much less molecules, just those, which are sitting on the hot spots, are involved in the SERS process at a very high enhancement level. The SERS spectra show lines in the region of 1000, 1200, $1600 \mathrm{~cm}^{-1}$, which originate from [Met]enkephalin but also other Raman lines from impurities, introduced to the colloidal silver particles due to the chemical preparation process. It has been shown that citrate forms a surface layer at the citrate reduced silver colloid, where the final citrate layer is oriented and bound differently 


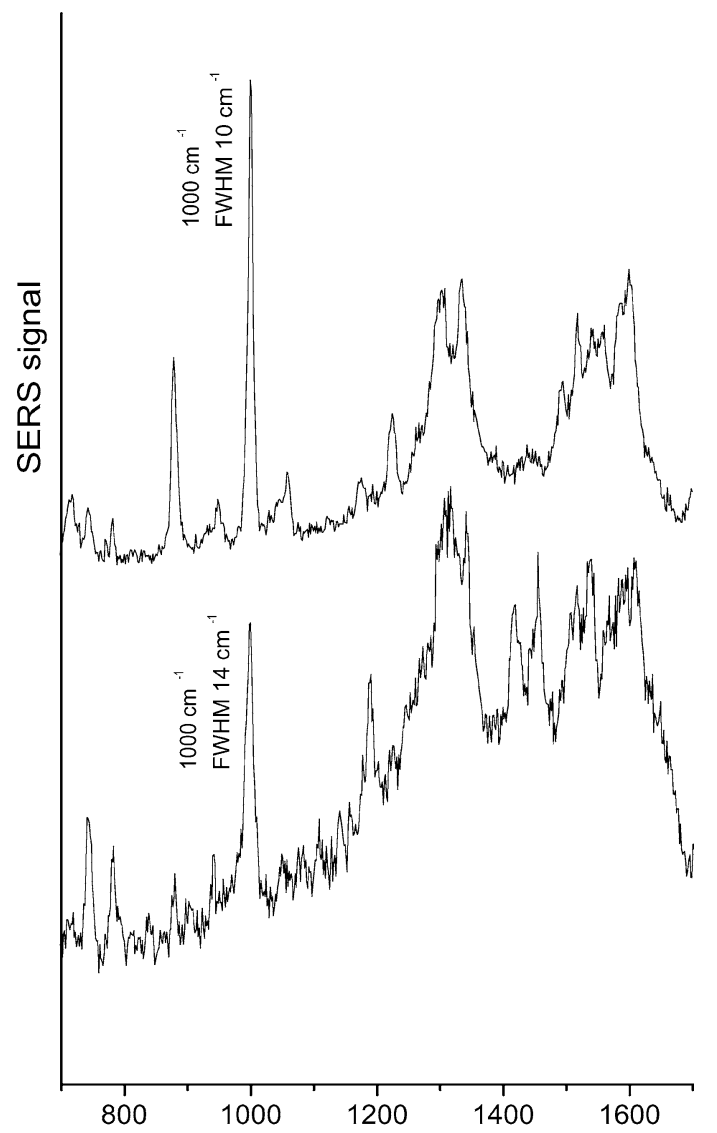

(a)

Raman Shift [ $\left.\mathrm{cm}^{-1}\right]$
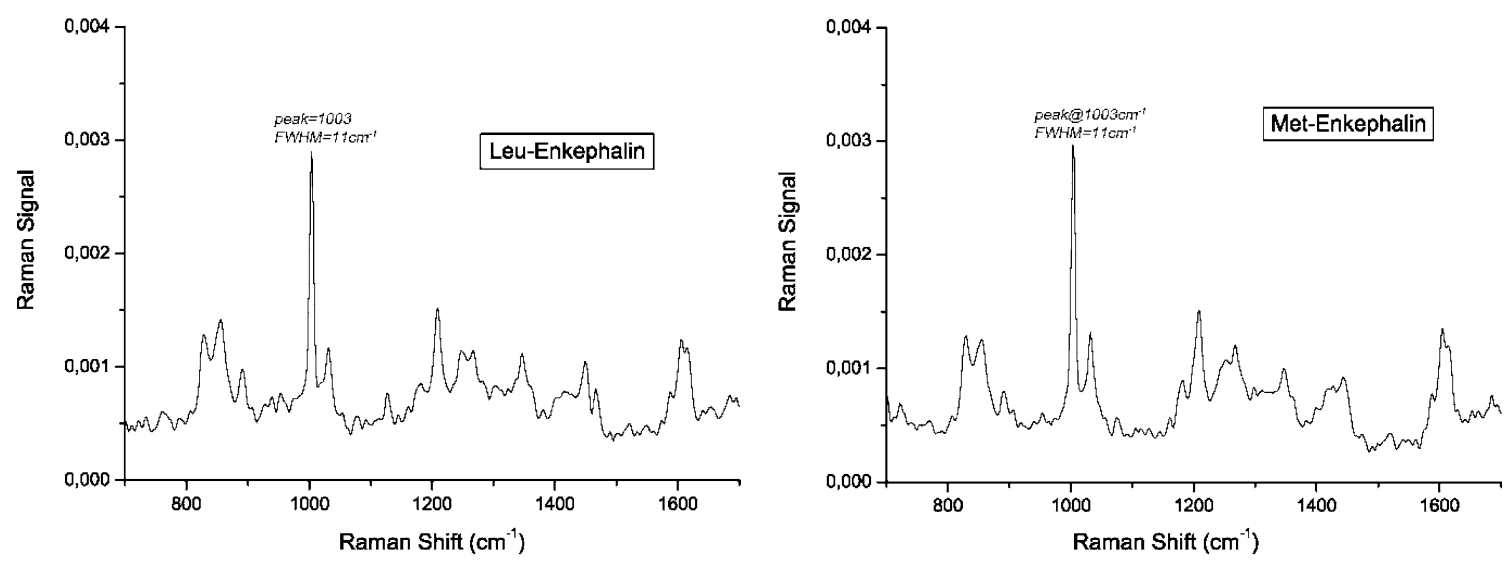

Fig. 2. (a) Unprocessed SERS spectra of [Met]enkephalin on silver colloidal clusters measured at two places of the sample, where the number of enkephalin molecules in the $1 \mu \mathrm{m}^{2}$ laser spot was on the order of 1000. (b) Normal Raman spectra of [Leu]enkephalin and [Met]enkephalin in $10^{-2} \mathrm{M}$ solution. (c) Structural formulas of [Leu]enkephalin and [Met]enkephalin. 

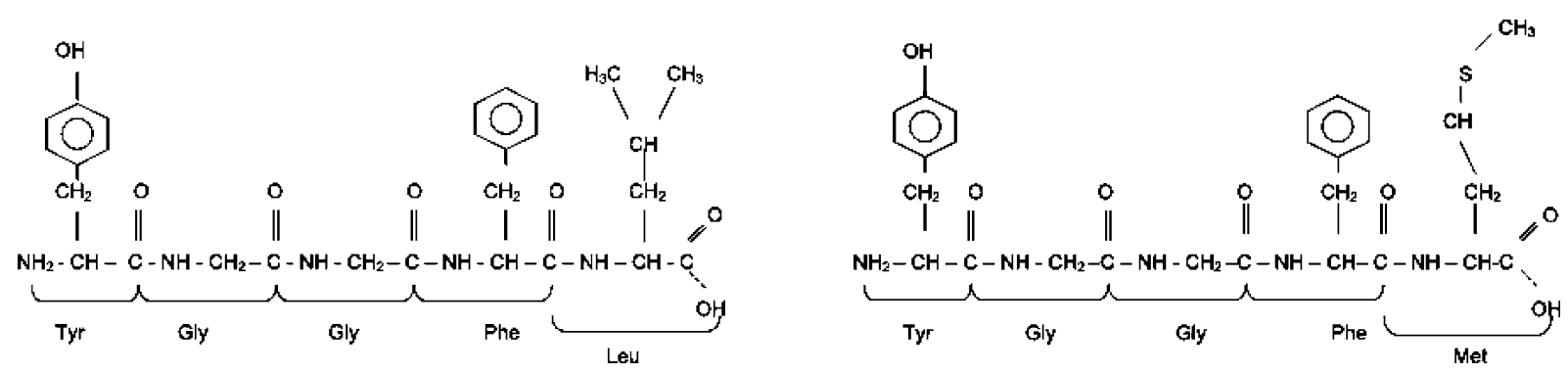

(c)

Fig. 2. (Continued.)

to excess citrate on the silver surface resulting in differences in the SERS spectra of citrate [15]. These citrate spectra always overlap with the spectra of the target molecule as it is also shown in Fig. 2a.

Compared to normal Raman spectroscopy on [Met]- and [Leu]enkephalin, where a fluorescence background exists at $514 \mathrm{~nm}$ excitation and Raman spectra were therefore measured at NIR excitation, the most SERS spectra do not show a measurable fluorescence background. This indicates that most of the molecules are in contact with the silver, which provides efficient nonradiative decay channels resulting in a strong fluorescence quenching.

The ring breathing mode of phenylalanine at $1000 \mathrm{~cm}^{-1}$ is the strongest line in the SERS spectra of [Met]enkephalin, which is in agreement with general experience in SERS that $\pi$-electrons strongly favor SERS enhancement. For example, SERS was discovered with the ring breathing mode of pyridine [16-18].

The $1000 \mathrm{~cm}^{-1}$ Raman line in one of the two spectra in Fig. 2a clearly shows an inhomogeneous broadening, which can sometimes occur in SERS, when the molecules contributing to the Raman signal "see" different environments resulting in slightly different Raman shifts. The effect of inhomogeneous broadening should be differently pronounced at different areas of the sample, as it is demonstrated by the two spectra. The line widths measured in the two SERS spectra are 10 and $14 \mathrm{~cm}^{-1}$, respectively. $12 \mathrm{~cm}^{-1}$ line width has been measured in normal Raman experiments, where usually no inhomogeneous broadening exists as all molecules feel the same (solvent) environment. Therefore, the $10 \mathrm{~cm}^{-1}$ line width suggests that only very few or even one single enkephaline molecule are spectroscopically selected from the 1000 molecules, which are present in the laser spot.

In Fig. 3, the concentration of the target molecule was reduced by 3 orders of magnitude compared to Fig. 2a. The concentrations of [Leu]enkephalin was $10^{-11} \mathrm{M}$ in the sample solution. When $5 \mu \mathrm{l}$ of this sample solution has been dried covering an area of approximately $2.5 \times 10^{-5} \mathrm{~m}^{2}$, this results in an average of one target molecule in the 1 micron laser focal spot. Raman signals of single [Leu]enkephalin molecules should appear when a single molecule meets a hot spot. Such situations were found by scanning over this sample. When a signal was found, measurements were performed on this fixed place of the sample in dependence on time.

Figure $3 \mathrm{a}$ shows the signal of the $1000 \mathrm{~cm}^{-1}$ mode measured in time sequence $(1$ second collection time each) within 120 seconds. The signal strengths show strong changes also when measuring from the same 1 micron spot. Figure 3a shows an example, where over the first 95 seconds no SERS signal was measured. Then, a SERS signal appears relatively abruptly, stays for about 20 seconds and vanishes again after this time. Such behavior, appearance of the $1000 \mathrm{~cm}^{-1}$ SERS signal within a 10-30 second time window has been observed in many measurements on single enkephalin molecules. Sudden 
(a)
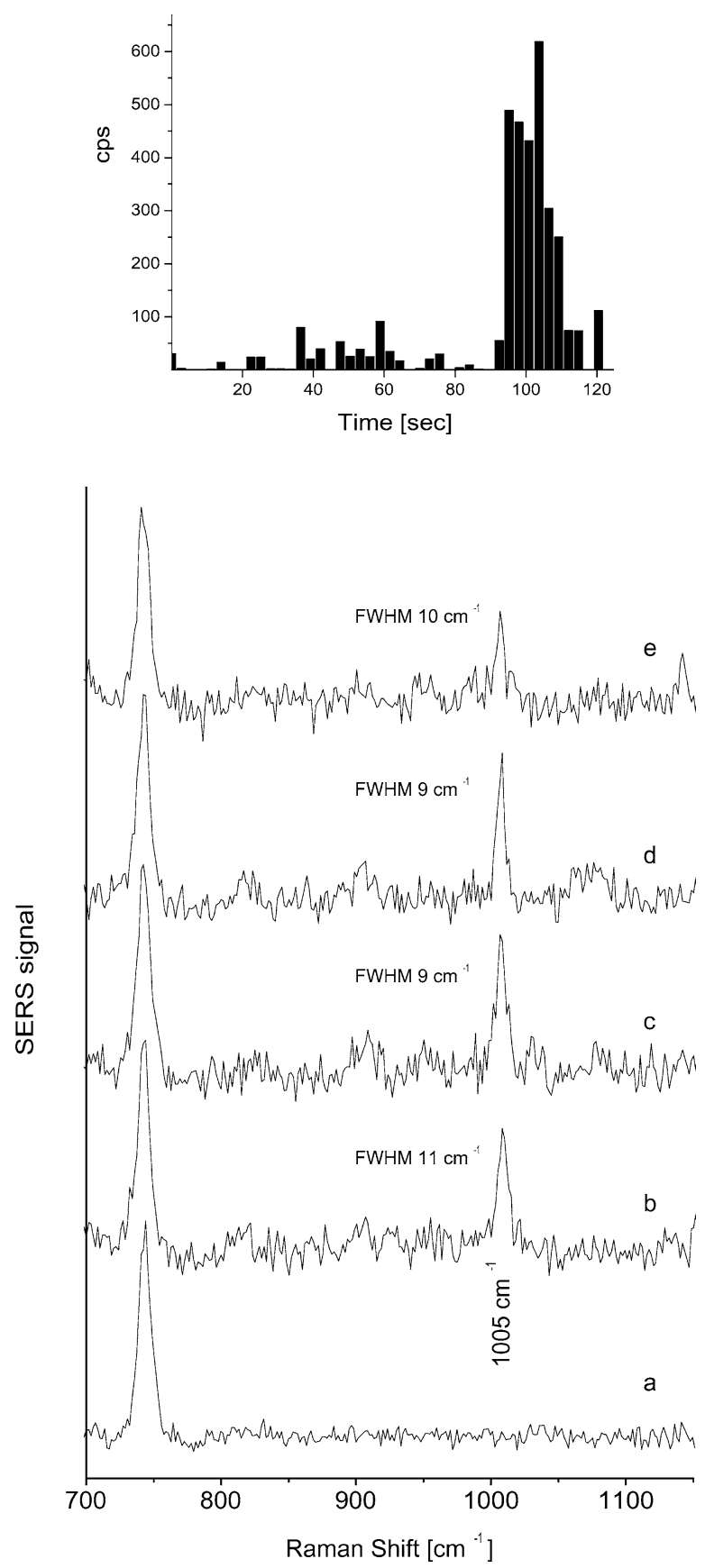

Fig. 3. SERS spectra of [Leu]enkephalin on silver clusters. The number of [Leu]enkephalin molecules in the $1 \mu \mathrm{m}^{2}$ laser spot size was on the order of one. (a) Time series of the $1000 \mathrm{~cm}^{-1}$ phenylalanine signal measured from one fixed spot on a sample with an average of one enkephalin molecule in the laser spot. Spectra were observed over a time interval of 120 seconds, 1 second collection time each. The signal level of $100 \mathrm{cps}$ represents approximately the background level. (b) Selected single molecule SERS spectra in a spectral window showing the $1000 \mathrm{~cm}^{-1}$ SERS line of phenylalanine and a line at $750 \mathrm{~cm}^{-1}$, which can be ascribed to citrate on the silver colloids. Spectra b)-e) were measured between 95 and 110 seconds on the time scale, spectrum a) was measured just before. 
spectral shifts and changes in scattering power, which appears as "blinking" of SERS signals has been reported by several authors. In the first time, it was claimed as evidence for single molecule detection. In the meantime, it became evident that this behavior is not necessarily connected to a single molecule SERS experiment and the effect has been also observed in lower concentration "many-molecule" SERS spectra [6,19-21]. In our case, there are no spectral changes, the $1000 \mathrm{~cm}^{-1}$ phenylalanine Raman line just appears and goes away vs time. For comparison, the signal at $750 \mathrm{~cm}^{-1}$, which can be ascribed impurities, always appears at almost the same signal level. A possible explanation for this observation is that a single [Leu]enkephalin molecule is diffusing on the colloidal silver cluster and can be "seen" only when it enters a hot spot [22]. Based on our measurements of the "on-times" of the SERS signal, the dwell time of a single [Leu]enkephalin molecule in a hot spot might be between 10 and 30 seconds.

Figure $3 \mathrm{~b}$ shows selected single molecule SERS spectra measured within the time interval shown in Fig. $3 \mathrm{a}$ in a spectral window, which displays the $1000 \mathrm{~cm}^{-1}$ SERS line of phenylalanine and a line at about $750 \mathrm{~cm}^{-1}$, which can be ascribed to an citrate impurity on the silver colloids [15]. Spectra b)-e) were measured in the time window between 95 and 115 seconds, spectrum a) shows a situation, when the $1000 \mathrm{~cm}^{-1}$ SERS signal does not exceed the noise level.

The spectra in Fig. $3 \mathrm{~b}$ show the expected result that inhomogeneous broadening in SERS spectra vanishes when single molecule Raman spectra are measured. The line widths measured for these single molecule spectra is $9-10 \mathrm{~cm}^{-1}$, little narrower than line widths measured in "normal" Raman scattering.

In summary, we have demonstrated that the SERS signal of the strongly enhanced ring breathing vibration of phenylalanine can be used as "intrinsic marker" for detecting a single enkephalin molecule and for monitoring its diffusion on the surface of the silver colloidal cluster without using a label. This result suggests the use of the phenylalanine $1000 \mathrm{~cm}^{-1}$ SERS line as spectroscopic signature for monitoring peptides and proteins containing this amino acid as a building block. Evidence for single molecule detection comes from concentration-volume/surface estimates, which result in an average of one target molecule in the focal area of the excitation laser. A second indication for single molecule measurements is a decrease in line width of the SERS line compared to inhomogeneously broadened "many molecule" SERS spectra.

\section{References}

[1] R. Rigler, M. Orrit and T. Basche, eds, Single Molecule Spectroscopy, Nobel Conference Lectures, Springer Verlag, Heidelberg, 2002.

[2] X. Michalet and S.C.R. Weiss, Physique 3 (2002), 619-644.

[3] K. Kneipp, H. Kneipp, I. Itzkan, R.R. Dasari and M.S. Feld, Journal of Physics 2 C14 (2002), R597.

[4] K. Kneipp, H. Kneipp, I. Itzkan, R.R. Dasari and M.S. Feld, Chemical Reviews 99 (1999), 2957.

[5] K. Kneipp, Y. Wang, H. Kneipp, L.T. Perelman, I. Itzkan, R.R. Dasari and M.S. Feld, Phys. Rev. Lett. 78 (1997), 1667.

[6] S. Nie and S.R. Emory, Science 275 (1997), 1102.

[7] K. Kneipp, Y. Wang, H. Kneipp, I. Itzkan, R.R. Dasari and M.S. Feld, Phys. Rev. Lett. 76 (1996), 2444.

[8] J. Hughes, T.W. Smith, H.W. Kosterlitz, L.A. Fothergill, B.A. Morgan and H.R. Morris, Nature 258 (1974), 577.

[9] L.A. Nafie, P.L. Prasad, H. Khouri and E. Doorly, Polymer Preprints 20 (1979), 85.

[10] S.L.-Han, E.R. Stimson, F.R. Maxfield and H.A. Scheraga, Int. J. Pept. Protein Res. 16 (1980), 173.

[11] S. Abdali, P. Refstrup, O. Faurskov Nielsen and H. Bohr, Biospectroscopy 72 (2003), 318.

[12] P.C. Lee and D. Meisel, Journal of Physical Chemistry 86 (1982), 3391.

[13] M.I. Stockman, V.M. Shalaev, M. Moskovits, R. Botet and T.F. George, Phys. Rev. B 46 (1992), 2821.

[14] V.A. Markel, V.M. Shalaev, P. Zhang, W. Huynh, L. Tay, T.L. Haslett and M. Moskovits, Phys. Rev. B 59 (1999), 10903.

[15] C.H. Munro, S.M. Smith, M. Garner, J. Clarkson and P.C. White, Langmuir 11 (1995), 3712.

[16] M. Fleischman, P.J. Hendra and A.J. McQuillan, Chem. Phys. Lett. 26 (1974), 123.

[17] D.L. Jeanmaire and R.P.V. Duyne, J. Electroanal. Chem. 84 (1977), 1.

[18] M.G. Albrecht and J.A. Creighton J. Am. Chem. Soc. 99 (1977), 5215. 
[19] A.M. Michaels, M. Nirmal and L.E. Brus, Journal of the American Chemical Society 121 (1999), 9932.

[20] A. Weiss and G. Haran, Journal of Physical Chemistry B 105 (2001), 12348.

[21] C.J.L. Constantino, T. Lemma, P.A. Antunes, P. Goulet and R. Aroca, Applied Spectroscopy (2003).

[22] K. Kneipp, H. Kneipp, P. Corio, S.D.M. Brown, K. Shafer, J. Motz, L.T. Perelman, E.B. Hanlon, A. Marucci, G. Dresselhaus and M.S. Dresselhaus, Phys. Rev. Lett. 84 (2000), 3470. 


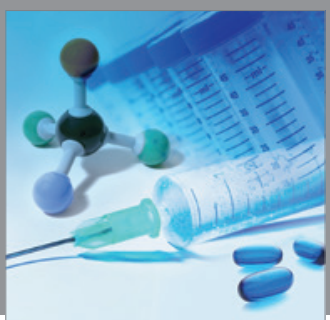

International Journal of

Medicinal Chemistry

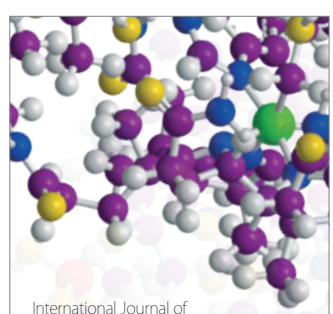

Carbohydrate Chemistry

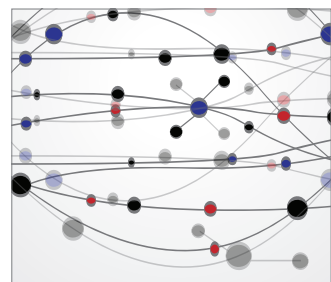

The Scientific World Journal
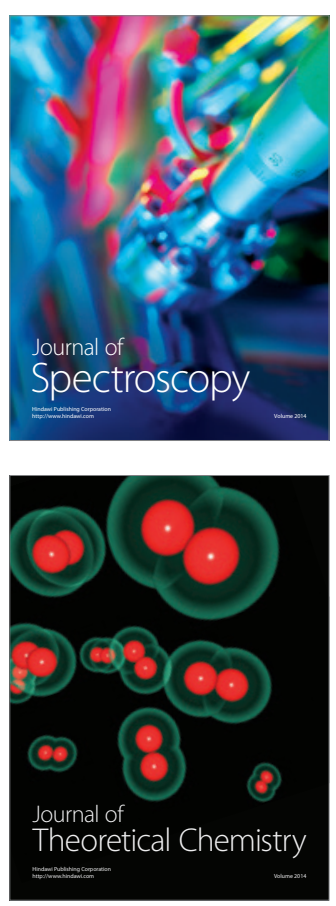
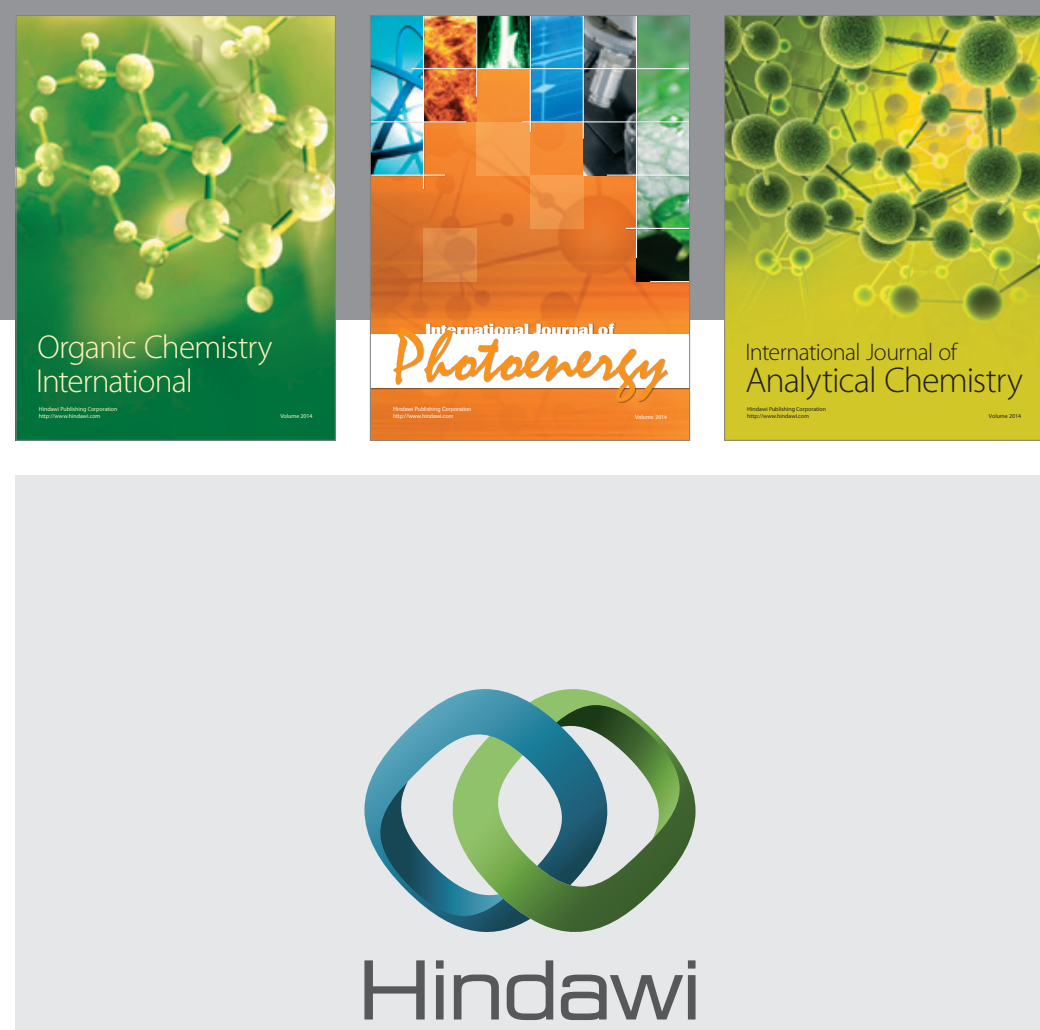

Submit your manuscripts at

http://www.hindawi.com
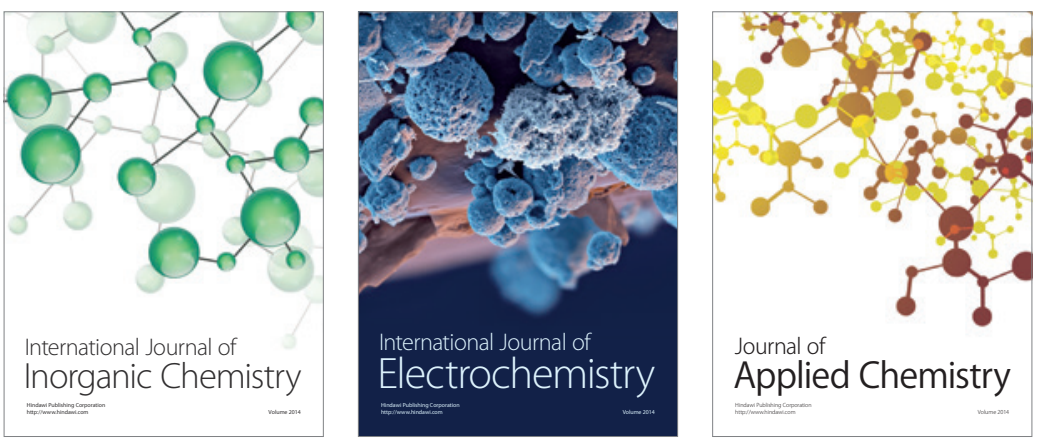

Journal of

Applied Chemistry
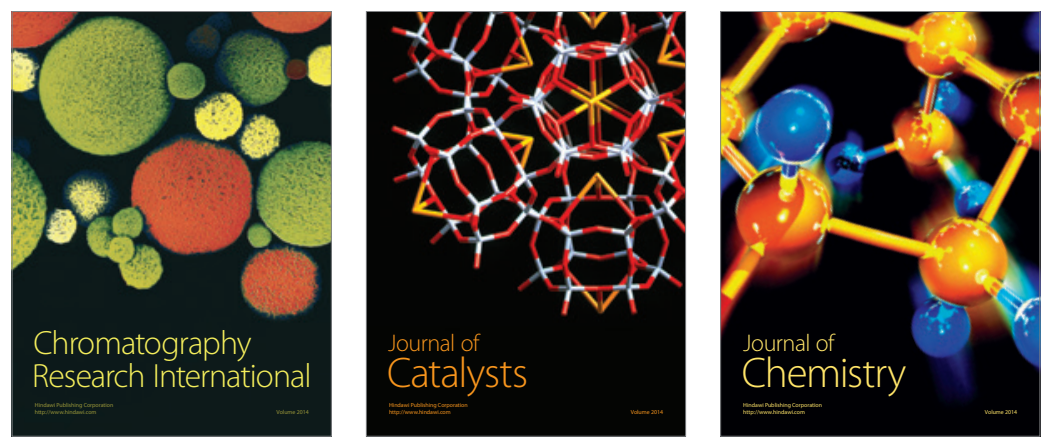
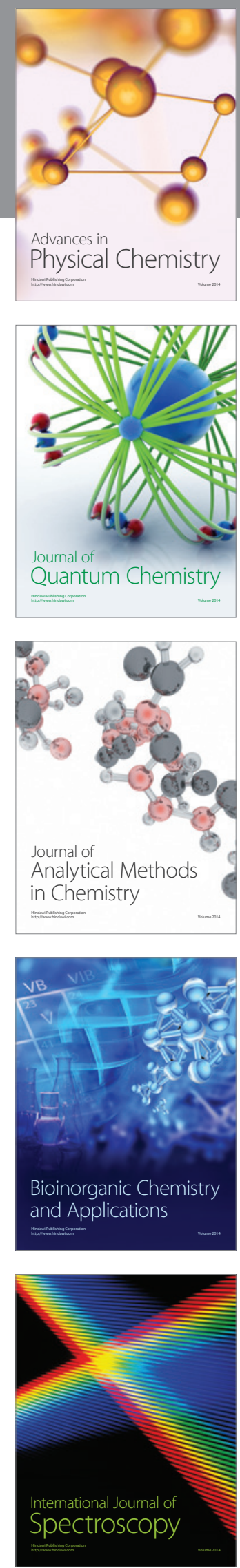